\title{
Politique
}

Politique

\section{L’autel du libéralisme : une revue des rapports Fortier, Gobeil et Scowen}

Johanne Bergeron

Numéro 11, hiver 1987

L’État privé

URI : https://id.erudit.org/iderudit/040549ar

DOI : https://doi.org/10.7202/040549ar

Aller au sommaire du numéro

Éditeur(s)

Société québécoise de science politique

ISSN

0711-608X (imprimé)

1918-6584 (numérique)

Découvrir la revue

Citer cette note

Bergeron, J. (1987). L'autel du libéralisme : une revue des rapports Fortier,

Gobeil et Scowen. Politique, (11), 129-138. https://doi.org/10.7202/040549ar d'utilisation que vous pouvez consulter en ligne.

https://apropos.erudit.org/fr/usagers/politique-dutilisation/ 


\section{L'autel du libéralisme: Une revue des rapports Fortier, Gobeil et Scowen}

Dans le creux de l'actualité politique et économique estivale, la publication de trois documents gouvernementaux ${ }^{1}$ venait secouer la moiteur ambiante. Il s'agissait des rapports Fortier sur la privatisation, Gobeil sur l'organisation gouvernementale, Scowen sur la déréglementation. Ces documents se ressemblent non pas tant à cause de la coïncidence temporelle de leur conception et de leur publication, mais parce qu'ils partagent une vision de l'État, un fondement idéologique commun. Efficacité économique, liberté, concurrence, rentabilité: tels sont en effet les maîtres mots. Le vent du libéralisme vient de souffler sur l'État québécois.

Le rapport Gobeil résume bien le postulat général qui rassemble les trois documents:

(...) le développement économique se fait de façon beaucoup plus saine et solide s'il s'appuie sur les forces du marché et (...) l'État y contribue de façon plus durable lorsqu'il crée les conditions et un environnement favorables au développement et à la croissance de l'entreprise. (p. 36)

Dans cette perspective, la réglementation entrave les libres forces du marché et la concurrence, elle accroît les coûts et les prix, diminue l'investissement et l'emploi, déresponsabilise les individus (Scowen, p. 14-15). Les sociétés d'État constituent une extension majeure de l'activité gouvernementale dans les sphères commerciales et industrielles, aux dépens du secteur privé et en concurrence avec lui (Fortier, p. vi). Les organismes gouverne- 
mentaux trop nombreux et souvent inefficaces étendent la place du secteur public dans la vie économique et sociale, contribuent à accroître le déficit budgétaire et induisent une fiscalité lourde et non concurrentielle (Gobeil, p. 26).

Ainsi convient-il maintenant d'alléger la réglementation et éliminer celle qui a des effets dysfonctionnels sur les décisions privées. Il faut réexaminer les entreprises publiques et privatiser celles dont le mandat peut être pris en charge de façon plus efficace par le secteur privé. Enfin, il faut rétablir l'équilibre budgétaire en réduisant les dépenses par une gestion plus rigoureuse des programmes gouvernementaux.

Tel est l'esprit de groupe qui rassemble les trois documents. Leur articulation s'effectue donc au niveau de la finalité poursuivie, qui revient en dernière instance à «redéfinir le rôle de l'État et tracer de nouvelles frontières entre secteurs public et privé» (Scowen, p. 5).

Le mandat du groupe de travail sur la déréglementation consistait à

(...) formuler des recommandations destinées à placer les entreprises du Québec dans une situation au moins aussi avantageuse que celles de l'Ontario en ce qui concerne l'ensemble des conditions réglementaires qui affectent leur productivité. (p. 1)

La réglementation est entendue au sens large, comme «toute intervention gouvernementale dans le fonctionnement et la conduite des activités privées», dont l'objet est de substituer aux indications et aux incitations du libre marché des directives ou des normes élaborées par les pouvoirs publics, afin de promouvoir des objectifs d'ordre socio-économique (p. 8). Si le groupe de travail se garde bien de mettre en cause la nécessité même de l'intervention de l'État, il n'en est pas moins convaincu a priori que tout accroissement de l'intervention publique «risque de faire plus de mal que de bien par rapport à l'objectif recherché» (p. 5); ainsi convient-il de réduire le poids de la réglementation de façon à rendre la 
société québécoise concurrentielle sur le plan économique, et d'ajuster le rôle de l'État en fonction de cet objectif.

Les réformes proposées s'inscrivent à trois chapitres principaux. En premier lieu, le rapport propose de nouveaux outils de contrôle du processus de réglementation. Paradoxalement peut-être, il recommande la création d'une nouvelle structure administrative, le Conseil de la législation et de la réglementation, qui serait chargé de la planification, de l'évaluation et du contrôle de l'ensemble des interventions gouvernementales de législation et de réglementation. Chaque ministère ou organisme de l'État serait tenu de soumettre à ce Conseil son plan annuel ou bi-annuel de législation et de réglementation pour examen et approbation.

En second lieu, le rapport se penche sur la réglementation sociale. Le diagnostic est clair: le régime réglementaire québécois est dans son ensemble plus lourd et plus coûteux que celui de l'Ontario. En conséquence, les recommandations visent notamment la réforme du régime de santé et sécurité au travail, l'abolition des décrets d'extension des conventions collectives, l'établissement d'une correspondance entre le niveau du salaire minimum et les normes de travail du Québec et de l'Ontario, une restriction de la portée des mesures anti-briseurs de grève, et enfin, en collaboration avec le secteur privé, l'établissement d'un plan de déréglementation en priorité dans les secteurs où les produits font l'objet de commerce interprovincial ou international.

Enfin, la réglementation économique est abordée, par l'examen de trois secteurs. Le groupe de travail recommande de déréglementer totalement l'industrie de la construction, de façon à y rétablir la concurrence et la liberté d'accès, que ce soit au niveau de l'embauche, de la syndicalisation ou des conditions de travail. De même, les services professionnels doivent être déréglementés, par la limitation de l'exclusivité des champs de pratique, la réévaluation du mécanisme de délégation des actes professionnels, l'abolition du pouvoir de fixer les tarifs d'honoraires, la libéralisation des règles 
relatives à la publicité. On recommande de la même façon la déréglementation totale de l'industrie du camionnage général et en vrac et du transport interurbain par autobus, au chapitre des tarifs et des permis.

Les préoccupations liées à la croissance du secteur public et au déficit budgétaire sont à la base des recommandations du rapport Gobeil, bien que ces questions n'y soient pas abordées explicitement. Ce rapport se compose de deux parties principales. La première partie, sur l'organisation gouvernementale, constitue un immense coup de balai dans les organismes publics. En caricaturant à peine, on peut la présenter comme une longue liste de comités, de commissions ou de conseils à maintenir, à réaménager ou, surtout, à abolir. Ce qui est gênant, c'est que n'apparaît aucune analyse qui justifierait la pertinence des choix posés. Ainsi par exemple, le Conseil consultatif sur l'environnement, le Conseil de la science et de la technologie, l'OPDQ, l'IQRC, la Régie du logement, devraient être abolis. Les responsabilités de cette dernière, de même que la société de la Place des Arts, le Musée des BeauxArts, le Musée d'art contemporain, l'Opéra de Montréal, devront être transférés au niveau municipal. Cela pourra certes entraîner une réduction des dépenses provinciales mais ne fait que reporter le problème sur le dos des municipalités, et il est loin d'être certain que cette solution conduise à une baisse globale des coûts.

La seconde partie porte sur la gestion des programmes publics. Ceux-ci sont regroupés par thème: santé, services sociaux et éducation, développement économique, main-d'œuvre et sécurité du revenu. En matière de santé et d'éducation, l'idée majeure du rapport Gobeil réside dans l'application de la décentralisation de gestion au niveau des établissements (hôpitaux et écoles) qui auraient désormais la responsabilité d'administrer leurs propres ressources financières et humaines.

Deux recommandations constituent des innovations radicales dans le contexte québécois. Aux niveaux primaire, secondaire et 
éventuellement collégial, les écoles se financeraient à l'aide de bons, d'une valeur égale à ce que coûte annuellement la formation d'un élève, que remettrait le gouvernement à chaque famille; les familles acquitteraient leurs frais de scolarité à l'aide de ces bons d'étude auprès de chacun des établissements fréquentés et seraient libres de les compléter par une contribution personnelle supplémentaire dans le cas où une école, privée, exigerait des frais additionnels. L'idée n'est rien de moins que révolutionnaire. Elle conduit à une espèce hybride de "privatisation publique» du système d'éducation: modèle de gestion privée et autonome des revenus et dépenses, financement de base public.

D'autre part, le rapport Gobeil recommande la privatisation totale des hôpitaux de taille petite et moyenne, ce qui va nettement à l'encontre de l'esprit des services de santé au Québec. Il recommande aussi d'inclure la valeur annuelle des soins de santé reçus par chaque contribuable à son revenu imposable, l'abolition des C.R.S.S.S., de même que le transfert des CLSC aux municipalités pour éliminer le doublement des services déjà dispensés par les hôpitaux. Cette dernière proposition est étonnante: sachant l'engorgement dont souffrent les cliniques externes des centres hospitaliers, ne serait-il pas logique au contraire de détourner au profit des CLSC une partie des services offerts? Il faudra sans doute attendre le rapport de la Commission Rochon pour une analyse éclairée et approfondie de ces questions.

Au chapitre du développement économique, la norme de référence demeure la liberté des forces du marché. Aussi le rapport critique-t-il sévèrement les diverses formes d'aide à l'industrie (subventions, prêts et garanties de prêts) et recommande la révision des programmes d'aide à l'entreprise, l'abolition des programmes de la SDI, mais le maintien des SPEQ et du REA. En matière de santé et sécurité au travail, le rapport se fait bref: il propose la réforme du régime actuel, ainsi que l'adoption de normes gouvernementales et la création d'un organisme chargé de les faire 
respecter. Les membres du groupe de travail sur la déréglementation protesteront-ils?... Enfin, plutôt que de poursuivre les programmes actuels d'aide sociale qui désincitent au travail, le rapport reprend l'idée de création d'un programme de garantie de revenu pour les bénéficiaires de l'aide sociale aptes au travail.

Le Comité sur la privatisation des sociétés d'État répondait au mandat de

conseiller le ministre (...) sur l'à-propos de transférer au secteur privé, en partie ou en totalité, les sociétés d'État dont les activités sont de nature commerciale ou industrielle. (p. i)

Son rapport regroupe les sociétés d'État en trois catégories et présente ses recommandations pour chacun des trois groupes: les entreprises stratégiques, qui, comme instruments d'intervention étatique, œuvrent dans des secteurs en concurrence avec le secteur privé, les monopoles publics qui, dit le rapport, "pourraient être exploités par le secteur privé» (p. 10), et les entreprises subventionnées dont l'objet est de gérer une activité subventionnée par le gouvernement.

Les recommandations sont faciles à énoncer. L'ensemble des dix entreprises stratégiques devront être privatisées. Elles cumulent les déficits, les objectifs ou les problèmes socio-économiques qui ont présidé à leur création sont aujourd'hui dépassés, le secteur privé pourrait prendre la relève de façon plus efficace. Le statut des sept monopoles publics devra faire l'objet d'une remise en question profonde, mais le rapport ne fait pas de leur privatisation une recommandation officielle. Il en est de même pour la trentaine d'entreprises subventionnées.

L'essentiel du rapport Fortier réside non pas tant dans ses recommandations que dans l'analyse proposée de la situation des sociétés d'État au Québec. Il rappelle pertinemment au sujet du débat sur leur impact économique que la plupart de ces sociétés œuvrent dans des secteurs à maturité ou en déclin, liés aux ressources naturelles (Hydro-Québec, SOQUEM, SOQUIP, REXFOR, SNA) 
ou à l'agro-alimentaire (SOQUIA, Madelipêche); aucune intervention publique n'a eu lieu dans des secteurs de pointe plus structurants sur l'économie (informatique, électronique ou même finances). Son questionnement central est également fort pertinent: les sociétés d'État actuelles répondent-elles aux objectifs d'intérêt public qui les sous-tendent? Leur performance engendre-t-elle les retombées économiques ou sociales maximales auxquelles l'État et les citoyens sont en droit de s'attendre?

Non, répond le rapport Fortier, qui voit alors dans la privatisation l'unique solution au problème. C'est oublier que le secteur public s'est constitué au Québec «à côté» du secteur privé, dans les espaces économiques laissés vacants. Rien n’indique alors, comme il est postulé ici, que le retrait de l'État serait compensé par une participation au moins équivalente du secteur privé. En outre, il est utopique de prétendre que l'absence de concurrence dans certains secteurs nationalisés, qui hausse les prix et réduit la qualité des biens ou services offerts, serait nécessairement corrigée par la privatisation. Même la théorie économique libérale admet l'idée qu'il existe des cas où le monopole géré publiquement représente la situation optimale (dans l'électricité, par exemple, où les rendements sont croissants).

Les objectifs qui ont présidé à la création des entreprises publiques et qui sont sous-jacents à leur fonctionnement actuel sont-ils périmés? La mission du «nation-building» apparaît pourtant comme une tâche fondamentale, de long terme, de tout État qui veut stimuler et orienter le développement économique; rien ne démontre que l'essor des entreprises québécoises et des cadres francophones soit une tâche accomplie, et qu'une relève «dynamique et abondante» (p. 29) se bouscule à l'entrée; le développement régional demeure un objectif qui ne sera jamais pris en charge par le secteur privé; les grands projets, profitables au niveau collectif, présentent des risques que peut difficilement assumer le secteur privé. 
Pourtant, affirme le rapport, les sociétés d'État sont déficitaires, leur performance est souvent décevante. Plutôt que de généraliser hâtivement, il convient d'examiner la performance des entreprises publiques cas par cas, à la lumière des objectifs qui leur sont assignés et des ressources dont elles disposent pour y parvenir; il faut également procéder à l'analyse des avantages et des inconvénients socio-économiques, des coûts et des retombées associés à leur activité, en termes de rentabilité sociale globale plutôt que purement financière.

Si les entreprises publiques tendent effectivement à utiliser leur pouvoir de marché pour accroître leur autonomie par rapport à l'État et au marché, il me semble que le problème en est alors un de gestion gouvernementale, et non pas inhérent par «nature» à la propriété publique elle-même. Ce n'est donc pas la société d'État à mettre en cause, mais bien l'inertie de l'État qui faillit à sa tâche fondamentale d'orientation et d'encadrement des choix de ses entreprises en fonction des objectifs stratégiques d'intérêt général qui constituent la raison d'être du secteur public. Il est probablement vrai que les entreprises publiques ont été sousutilisées par rapport aux ressources qu'elles contrôlent, à cause de ce manque de directives de la part des ministères de tutelle.

Il y a deux idées gênantes dans ce rapport. Les sociétés d'État seraient intrinsèquement, presque par nature, inefficaces; ces organisations, remises au secteur privé, dont «libérées des contraintes découlant de la formule de société d'État» (p. 6), deviendraient plus performantes et plus productives. Il s'agit là d'un à priori pour le moins discutable. Il existe certes des contraintes que doivent affronter ces entreprises, mais elles tiennent bien davantage à leur position dans leur secteur respectif d'activité et à leur mandat qu'à leur statut juridique.

La seconde idée semble partagée par les trois rapports discutés ici. Il s'agit du postulat qui veut que le secteur public s'oppose par essence au secteur privé, que leurs intérêts et leur fonctionnement 
soient antinomiques. L'économie est vue comme une sphère close où tout ce qui est public représente un espace en moins pour le secteur privé, accaparé à ses dépens. Or, au contraire, l'expérience québécoise de nationalisation montre un respect essentiel des lois du marché; elle ne s'est pas effectuée contre les intérêts privés, qui ont pu bénéficier largement des retombées associées aux activités publiques. Même la constitution d'Hydro-Québec, dont on dit qu'elle est un monopole, a laissé au secteur privé l'ensemble de l'électricité produite pour des fins industrielles, ce qui représentait en 1963 quelque $40 \%$ de la capacité installée totale.

Le parti-pris en faveur de l'économie privée s'explique peutêtre par la composition des groupes de travail qui ont réalisé les trois documents: les «sages» sont issus du secteur privé. Mais cela va plus loin. L'idéologie libérale connaît un sursaut de popularité dans les économies occidentales et notamment au Québec, ce qui éclaire le recours à des gestionnaires privés d'expérience pour conseiller le Prince. Mais il serait bête que par amour d'une idéologie les décideurs publics fassent l'économie d'une analyse approfondie des objectifs et du fonctionnement des sociétés d'État, des organismes publics et de la réglementation à la lumière des objectifs et des besoins qui les ont suscitées, et balaient du revers de la main les acquis de la Révolution tranquille.

Il y a dans ces trois rapports suffisamment de matière pour susciter la colère des groupes sociaux touchés par les diverses recommandations et pour alimenter des débats houleux. Souhaitons que l'harmonie sociale ne soit pas sacrifiée sur l'autel du libéralisme économique.

$$
\text { ** }
$$

1. Comité sur la privatisation des sociétés d'État. De la Révolution tranquille.. à l'an deux mille. Rapport présenté à M. Pierre Fortier, ministre délégué à la privatisation. Québec, juin 1986. 
Groupe de travail sur la déréglementation. Réglementer moins et mieux. Présidé par $\mathbf{M}$. Reed Scowen. Québec, Les publications du Québec, juin 1986.

Groupe de travail sur la révision des fonctions et des organisations gouvernementales. Rapports. Présidé par M. Paul Gobeil. Québec, mai 1986.

Johanne Bergeron

Université de Montréal 